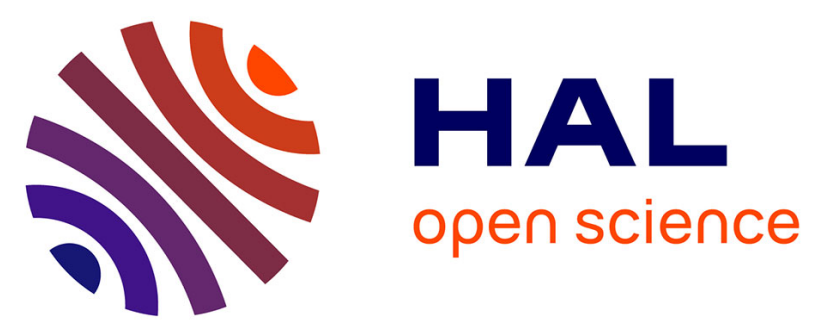

\title{
Binding of chloroplast signal recognition particle to a thylakoid membrane protein substrate in aqueous solution and delineation of the cpSRP43-substrate interaction domain
}

Peter Cain, Iris Holdermann, Irmgard Sinning, Arthur E Johnson, Colin Robinson

\section{To cite this version:}

Peter Cain, Iris Holdermann, Irmgard Sinning, Arthur E Johnson, Colin Robinson. Binding of chloroplast signal recognition particle to a thylakoid membrane protein substrate in aqueous solution and delineation of the cpSRP43-substrate interaction domain. Biochemical Journal, 2011, 437 (1), pp.149155. 10.1042/BJ20110270 . hal-00600762

\section{HAL Id: hal-00600762 https://hal.science/hal-00600762}

Submitted on 16 Jun 2011

HAL is a multi-disciplinary open access archive for the deposit and dissemination of scientific research documents, whether they are published or not. The documents may come from teaching and research institutions in France or abroad, or from public or private research centers.
L'archive ouverte pluridisciplinaire HAL, est destinée au dépôt et à la diffusion de documents scientifiques de niveau recherche, publiés ou non, émanant des établissements d'enseignement et de recherche français ou étrangers, des laboratoires publics ou privés. 


\title{
Binding of chloroplast signal recognition particle to a thylakoid membrane protein substrate in aqueous solution and delineation of the cpSRP43- substrate interaction domain
}

\author{
Peter Cain ${ }^{1}$, Iris Holdermann ${ }^{2}$, Irmgard Sinning ${ }^{2}$, Arthur E. Johnson ${ }^{3,4}$ and Colin Robinson ${ }^{1}$ \\ ${ }^{1}$ School of Life Sciences, University of Warwick, Coventry CV4 7AL, UK. \\ ${ }^{2}$ Biochemie-Zentrum der Universität Heidelberg, INF 328, D-69120 Heidelberg, Germany. \\ ${ }^{3}$ Department of Molecular and Cellular Medicine, Texas A\&M Health Science Center, College \\ Station, TX 77843-1114, USA. \\ ${ }^{4}$ Departments of Chemistry and of Biochemistry and Biophysics, Texas A\&M University, \\ College Station, TX 77843-114, USA.
}

Correspondening author: Colin Robinson. Telephone: 44-2476-523557; FAX: 44-2476-523568;

E-mail: colin.robinson@warwick.ac.uk

Short title: Dissection of the cpSRP43-Lhcb1 interaction

\begin{abstract}
Synopsis
A chloroplast signal recognition particle (cpSRP) comprising cpSRP54 and cpSRP43 subunits mediates the insertion of light-harvesting proteins into the thylakoid membrane. We dissected its interaction with a full length membrane protein substrate in aqueous solution by insertion of sitespecific photoactivatable crosslinkers into in vitro-synthesised Lhcb1. We show that Lhcb1 residues 166-176 crosslink specifically to the cpSRP43 subunit. Some crosslink positions within Lhcb1 are in the 'L18' peptide required for targeting of cpSRP substrates, while other crosslinking positions define a new targeting signal in the third transmembrane span. Lchb1 was not found to crosslink to cpSRP54 at any position, and crosslinking to cpSRP43 is unaffected by the absence of cpSRP54. cpSRP43 thus effectively binds substrates autonomously, and its ability to independently bind an extended, 20+-residue substrate region highlights a major difference to other SRP types where the SRP54 subunit binds to hydrophobic target sequences. The data also show that cpSRP43 can bind to a hydrophobic, 3-membrane span substrate in aqueous solution, presumably reflecting a role for cpSRP in the chloroplast stroma. This mode of action, and the specificity of the cpSRP43-substrate interaction, may be associated with cpSRP's uniquely posttranslational mode of action.
\end{abstract}

Key words: SRP, LHC, photosystem II, Lchb1, thylakoid membrane, chloroplast

\footnotetext{
Abbreviations: cpSRP, chloroplast signal recognition particle; LHCP, light-harvesting chlorophyll-binding protein; Lhcb1, major light-harvesting chlorophyll-binding protein of photosystem II.
} 


\section{Introduction}

Signal recognition particle (SRP) is a ubiquitous targeting factor that plays a pivotal role in the translocation of proteins into and across membranes. In eukaryotes, SRP is a particle comprising a 7S RNA molecule and 6 protein subunits, including a $54 \mathrm{kDa}$ subunit (SRP54) that binds the hydrophobic signal peptides of nascent proteins that are targeted to the endoplasmic reticulum (reviewed in [1]). A more compact SRP carries out a similar function in bacteria; Escherichia coli SRP contains a single protein, Ffh (a homologue of SRP54) and a 4.5S RNA molecule. This complex interacts with FtsY to insert the substrate into the plasma membrane with the assistance of membrane-bound machinery including YidC [2]. As in eukaryotes, membrane proteins are inserted co-translationally.

Chloroplasts also contain an SRP pathway for the targeting of membrane proteins, primarily used for the insertion of a family of abundant thylakoid membrane proteins, namely light-harvesting chlorophyll-binding proteins (LHCPs) of the photosystem I and II light-harvesting complexes (reviewed in [3]). These are synthesized in the cytosol, imported into the chloroplast stroma and then inserted into the thylakoid membrane by a pathway that involves cpSRP, cpFtsY and a membrane-bound homologue of bacterial YidC, termed Alb3 [4-6]. Although clearly related to bacterial SRP pathways, the chloroplast SRP (cpSRP) pathway exhibits a number of unique characteristics. First, cpSRP acts post-translationally, in strict contrast to the obligatorily cotranslational systems outlined above. Secondly, cpSRP comprises two subunits, cpSRP54 and cpSRP43, and lacks an RNA molecule. cpSRP54 is homologous to the SRP54 subunits from other SRP systems, but the cpSRP43 subunit is unique to cpSRP [7]. Its presence appears to be connected with the unexpectedly narrow substrate specificity of the chloroplast SRP pathway; whereas the vast majority of membrane proteins are targeted to the endoplasmic reticulum or bacterial plasma membrane by the SRP pathway, only a specific subset of highly abundant LHCPs use the post-translational chloroplast SRP pathway [8,9]. Surprisingly, the remaining thylakoid membrane proteins are inserted by a pathway that does not require any known form of protein targeting apparatus, including the Sec system, cpSRP, FtsY or Alb3 [10,11]. LHCP proteins contain a specific motif that directs them to the SRP pathway and away from the novel 'spontaneous' insertion pathway. This is a key 18-residue recognition element for cpSRP43, known as the L18 peptide, that is located between transmembrane (TM) spans 2 and 3 [12,13].

Studies on the structure and function of cpSRP have revealed several points. First, a heterodimeric combination of E. coli-expressed cpSRP43 and cpSPR54 is active in promoting Lhcb1 insertion into thylakoids in the presence of cpFtsY [14]. Secondly, it is believed that cpSRP43 contains several discrete domains including four sequential ankyrin repeats [15] located between residues 130 and 254 [16]. These ankyrin repeats (ANK 1-4) have been implicated in protein-protein interactions and are likely to be involved in complex formation. The second repeated structures are chromodomains. Two of these domains (CD2 and 3) are located between residues 271 to 368 at the $\mathrm{C}$-terminus of cpSRP43 [16] and a third chromodomain (CD1) is located between residues 24 and $76 \mathrm{~N}$-terminal to the ankyrin repeats [3]. The structures of all three chromodomains have been solved using triple resonance NMR experiments [17].

More recently, the crystal structure of cpSRP43 has been solved [18], and the cpSRP43 cocrystallised together with bound L18 peptide. This has provided the first information on the mode of interaction between cpSRP and its substrates. In this report we have sought to generate a 
detailed understanding of the cpSRP-Lchb1 interaction using used sensitive, site-specific crosslinkers to probe the nature of this interaction in detail, using full-length, insertion-competent substrate instead of purified L18 peptide. We show that cpSRP43 interacts with an extended region of Lhcb1 that includes residues from the L18 peptide and TM span 3. We also show that this highly specific interaction takes place with equal efficiency in the absence of cpSRP54, suggesting that cpSRP43 is effectively a soluble, stromal factor capable of binding incoming substrate molecules on its own.

\section{Experimental}

\section{Protein Expression and Purification}

Expression and purification of cpSRP43 and cpSRP54 from Arabidopsis thaliana was performed as described [18]. The construct of cpSRP43 with a C-terminal truncation consisting of CD3 (cpSRP43 279 ) was cloned into pETM11 (kind gift from Gunter Stier, EMBL Heidelberg, Germany). The cpSRP43-Y204A point mutation was generated in the $\mathrm{pET} 24 \mathrm{a}$ vector using the QuickChange system (Stratagene). A full-length cDNA clone encoding the precursor of Pea (Pisum sativum) Lhcb1 [19] was used as template to generate amber-stop-codon (TAG) mutations at the at the indicated amino acid positions.

\section{Preparation of suppressor $t R N A$ s and labelled protein, and crosslinking reactions} Lys-tRNA $^{\text {amb }}$ and $N^{\varepsilon}$-(5-azido-2-nitrobenzoyl)-Lys-tRNAamb ( $\varepsilon$ ANB-Lys-tRNA ${ }^{\text {amb }}$ ) were synthesized, aminoacylated, and modified as described before [20,21]. cDNAs were transcribed in vitro by SP6 polymerase followed by in vitro translation in a wheat germ lysate system in the presence of $\left[{ }^{35} \mathrm{~S}\right]$ methionine at $26^{\circ} \mathrm{C}$ for $1 \mathrm{~h}$ [22]. Amber codon suppression reactions included the addition of either Lys-tRNA ${ }^{\mathrm{amb}}$ or $\varepsilon A N B-L y s-t R N A^{\mathrm{amb}}$ as performed by McCormick et al. [21]. For cross-linking reactions, a modified wheat germ lysate system was used with glutathione in the place of DTT and with all procedures prior to photolysis performed under dim red safelight in darkroom conditions [23]. Immediately after translation, samples were dialysed for 2 hours into translation buffer [12 mM HEPES, pH7.5, $\left.80 \mathrm{mM} \mathrm{KOAc,} \mathrm{pH} \mathrm{7.5,} 2 \mathrm{mM} \mathrm{Mg}(\mathrm{OAc})_{2}\right]$. For crosslinking reactions, double volume reactions were prepared so that one half could be analysed directly by SDS-PAGE leaving the other half for Talon re-purification. Crosslinking reactions were made up to a total $100 \mu \mathrm{l}$ in $10 \mathrm{mM}$ Hepes-KOH (pH 8.0), $5 \mathrm{mM} \mathrm{MgCl}_{2}$ and contained 16 $\mu \mathrm{l}$ of translation product and $2.5 \mu \mathrm{g}$ of purified recombinant cpSRP or subunit thereof where required. Binding between Lhcb1 and cpSRP was performed at $26^{\circ} \mathrm{C}$ for 30 min and was immediately followed by placing the samples on ice for $10 \mathrm{~min}$ in preparation for photo-lysis. Samples were photo-1ysed on ice as in [21] but for 3 min using a $100 \mathrm{~W}$ mercury arc lamp (UVP) with a band pass filter (UVP) to isolate the UV emission at $365 \mathrm{~nm}$.

\section{Talon re-purification}

Post-crosslinking reactions were re-purified using the Talon affinity batch method. Talon resin was washed in 3 times in $20 \mathrm{mM}$ Tris- $\mathrm{HCl}(\mathrm{pH} 8.0), 400 \mathrm{mM} \mathrm{NaCl}, 5 \mathrm{mM}$ imidazole by centrifugation at $6000 \mathrm{x}$ g for $1 \mathrm{~min} .150 \mu \mathrm{l}$ of the $50 \%$ talon suspension was stirred into $50 \mu \mathrm{l}$ of the post-crosslinking sample. Binding was performed on ice for 1 hour with occasional stirring. Samples were then washed 6 times as before in $800 \mu 1$ of buffer, and the Talon resin was re-suspended in $30 \mu$ l elution buffer [20 mM Tris- $\mathrm{HCl}(\mathrm{pH} 8.0), 400 \mathrm{mM} \mathrm{NaCl}, 150 \mathrm{mM}$ imidazole] followed by SDS-PAGE sample buffer. 


\section{Structure figures}

Structure figures were generated with Pymol (http://www.pymol.org).

\section{Results}

Probing the interactions between Lhcbl and cpSRP

cpSRP interacts with a discrete set of substrates, but the precise contributions of the cpSRP43 and cpSRP54 subunits have not been determined. In other systems, the SRP54 subunit plays a dominant role in the binding of the targeting signals within substrates, and we expected that cpSRP54 would play an equally prominent role in binding newly-imported LHCP proteins, with cpSRP43 perhaps acting as an ancillary specificity factor. cpSRP43 has been shown to bind purified L18 peptide with high affinity [18] but no studies to date have characterised the binding of cpSRP to a full-length, insertion-competent substrate in aqueous solution. The substrate used in this study was Lhcb1, a major component of the photosystem II light-harvesting complex. This protein contains three trans-membrane spans, and the membrane-bound 3-dimensional structure is known in some detail [24].

We studied the binding of Lhcb1 to cpSRP subunits using a cross-linking strategy in which UV-photoactivatable cross-linkers were placed at specific positions within Lhcb1. The crosslinker is covalently attached to the Lys side chain of an aminoacylated amber suppressor tRNA (Lys-tRNA $^{\mathrm{amb}}$ ) producing EANB-Lys-tRNA $^{\mathrm{amb}}$. This suppressor tRNA only recognizes amber stop codons during protein synthesis, allowing for the uncharged photo-reactive probe to be inserted at specific residues in the polypeptide during in vitro transcription-translation, determined by site-specific mutagenesis of the cDNA template [21].

Fig. 1 shows the overall domain structure of Lhcb1 including the transit peptide (TP) that mediates chloroplast import, the three trans-membrane spans (TM1-3) and the L18 peptide (residues 152 to 169) just prior to TM3. Since cpSRP clearly interacts with the L18 region, we focused attention on this region plus the TM spans on either side (TM2 and TM3) with the crosslinker present in 17 different positions throughout this region as shown (positions are arrowed, with numbering from the start of the mature protein). Translation in the presence of EANB-Lys-tRNA ${ }^{\mathrm{amb}}$ leads to the presence of crosslinkers at these sites ( 1 site per experiment). After transcription and translation in vitro, translation mixtures were incubated with cpSRP43 and cpSRP54 alone, or in combination, in order to understand the individual contributions of these subunits to cpSRP-substrate interactions. We also used a truncated version of the cpSRP43, termed $43 \Delta$, in which the third chromodomain is deleted (see Experimental procedures).

Crosslinkers in Lhcb1 at positions 169 and 176 specifically react with cpSRP43 Lhcb1 translation products with single crosslinkers at the 17 indicated positions were tested for interaction with purified cpSRP (or individual subunits), after which the crosslinking reaction was initiated by UV light. Only those showing a positive crosslink are described in detail below, with the Lhcb1 variants denoted by Lhc\#\#\#, where \#\#\# represents the residue number. Some of the strongest crosslinks were obtained with Lhc169 and 176, and these data are shown in Fig. 2. Samples were either analysed directly ('total' samples; upper panel) or after passage through a Talon affinity resin to affinity-purify the His-tagged cpSRP plus any covalently bound Lhcb1 photoadduct (lower panel). 
Activation of the Lhc169 and 176 crosslinks in buffer alone (-) does not generate specific adducts, with only weak non-specific bands present in the $50 \mathrm{kDa}+$ region in either the total or Talon panels. The presence of cpSRP43 alone results in the generation of two adducts, of $63 \mathrm{kDa}$ and $76 \mathrm{kDa}$, while a single strong adduct of $69 \mathrm{kDa}$ is formed in the presence of cpSRP43 $\Delta$. The crosslinks to cpSRP43 are denoted X1 and X2, while the crosslink to cpSRP43 $\Delta$ is denoted X $\Delta$; all three are also indicated by asterisks for clarity. The same photoadducts are formed when SRP54 is also present, strongly indicating that the Lhcb1 variants do not crosslink with cpSRP54, but rather continue to crosslink to cpSRP43. These photoadducts re-purify with the cpSRP during Talon affinity chromatography and are thus believed to be generated by specific interaction between Lhcb1 proteins and cpSRP43. It is unclear why two crosslinked adducts to cpSRP43 are observed; this may indicate the existence of two different conformational states for the Lhcb1-cpSRP43 complex, or of two different crosslinking sites on cpSRP43 reached by the flexible probe that give photoadducts with different electrophoretic mobilities. Alternatively, the $\mathrm{X} 2$ adduct may be generated by proteolytic clipping of $\mathrm{X} 1$.

Essential controls are shown in Fig. 3. First, Lhc169 was synthesised with Lys present at position 169, rather than the cross-linker (D169K lanes), by including Lys-tRNA ${ }^{\mathrm{amb}}$ in the translation system instead of $\varepsilon A N B-L y s-t R N A^{a m b}$. The translation product was incubated with cpSRP43 $\Delta$ as above, and no cross-linked species was observed in either the presence or absence of UV light. Secondly, the $69 \mathrm{kDa}$ adduct is observed if the cross-linker was present and activated by UV light, but no adduct was generated in the absence of UV light (Lhc169 lanes). The adducts are thus generated by specific interaction between the photo-activated crosslinker and cpSRP43 $\Delta$.

\section{Interactions with SRP43 are clustered within the L18 peptide and TM3}

We routinely observed adducts to cpSRP43 when the crosslinker was present at residues 166, 169, 174 or 176 in pea Lhcb1. Fig. 4 effectively summarises the extent of the cpSRP43-Lhcb1 interaction region, as assessed using this technique. These constructs were incubated with buffer (-), cpSRP43 alone (43) or cpSRP43 $(43 \Delta)$. Activation of the cross-link again leads to the formation of specific $63 \mathrm{kDa}$ and $76 \mathrm{kDa}$ adducts in the presence of cpSRP43, or a $69 \mathrm{kDa}$ adduct if the cpSRP43 $\Delta$ variant is present. All four constructs routinely crosslink to cpSRP43 or the truncated form to yield a reproducible pattern of interaction. Fig. 4 also shows that no adducts are observed when the crosslinker is positioned at the opposing end of L18, at position 152, and we obtained identical results when the crosslinker was placed, at position 164, just outside of L18 at 150 and 148, or in TM2 (data not shown). No change in crosslinking pattern was observed when cpSRP54 was included in the reaction (data not shown) and we conclude that (i) residues 166176 are in intimate contact with SRP43, and (ii), cpSRP43 on its own contains all of the information required to interact strongly and specifically with Lhcb1.

A tyrosine 'hook' within cpSRP43 is essential for the binding of the L18 peptide, but not for binding of a second determinant.

Tyrosine 204 within the cpSRP43 structure was previously shown to be crucial in preserving interactions with purified L18 peptide, and we hypothesised that this residue provided a crucial hook-like structure on the binding face of cpSRP43 for interactions with Lhcb1 [18]. We characterised the interaction between the cpSRP43-Y204A mutant and full-length Lhcb1, in both the presence and absence of cpSRP54 (Fig. 5) using the Lhc169 construct. Here, the crosslinking probe is ideally positioned within L18 to probe this interaction and the importance of Tyr204. In 
control tests, the reaction mixture lacking cpSRP (-) exhibits neither of the $63 \mathrm{kDa}$ and $76 \mathrm{kDa}$ crosslinks that characterise binding to cpSRP43. These bands are, however, observed in the presence of cpSRP43 (43) or a combination of cpSRP43 and cpSRP54 (43/54). The major 76 $\mathrm{kDa}$ crosslink (X1) is indicated and both the $76 \mathrm{kDa}$ and $63 \mathrm{kDa}$ adducts are indicated by asterisks in Fig. 5. In the other control test, where cpSRP43 $\Delta$ is present $(43 \Delta)$, the usual $69 \mathrm{kDa}$ crosslink is detected (denoted by X $\Delta$ ). In the presence of the cpSRP43-Y204A mutant alone $\left(43^{*}\right)$, none of these crosslinks is detected, but we detect a band that runs just below the $76 \mathrm{kDa}$ adduct (denoted by arrowhead). This is consistent with crosslinking to the cpSRP43-Y204A mutant, and the slightly increased gel mobility of this adduct almost certainly stems from the fact that the Y204A mutant does not bear a C-terminal 6-His tag, while the non-mutated cpSRP43 does. Assuming that this band does represent the crosslinking of Lchb1 (position 169) to cpSRP43-Y204A, the interaction between the mutated cpSRP43 and Lhcb1 appears to be stabilised in the presence of cpSRP54, at least to an extent.

\section{Discussion}

cpSRP directs the targeting of a select group of LHCPs into the thylakoid membrane, using a mode of action that is entirely post-translational. cpSRP thus has a unique action among the SRP family, since the other forms are generally believed to act co-translationally, and this raises the question of how cpSRP binds newly-imported thylakoid membrane proteins so specifically in the chloroplast stroma. Previous studies have focused on the role of the L18 peptide as a specificity determinant, and this peptide has been shown to bind to a distinct groove within cpSRP43 [18]. However, a wealth of studies on other SRP molecules has pinpointed the cpSRP54 subunit as the key site for initial interaction with substrate targeting signals, which are invariably hydrophobic stretches of amino acids. Consistent with this idea, cpSRP54 has been shown to be capable of crosslinking to isolated TM span regions derived from thylakoid membrane proteins [25], again suggesting a role for this subunit as the initial site of interaction. In this study we have set out to identify sites of interaction between Lhcb1 and cpSRP subunits using a highly specific crosslinking approach. The crosslinkers were inserted into full-length Lhcb1 and this is the first time that the cpSRP-substrate interaction has been dissected using an authentic, full-length substrate. Moreover, the experiments have been carried out in aqueous solution, which we believe more accurately reflects the insertion process by which stromal intermediate forms integrate into thylakoids. Several of the constructs crosslink to cpSRP43 and the results provide information on key areas of the cpSRP mode of action. None of the constructs crosslink to cpSRP54, hence we are unable to shed light on the role of this subunit in substrate binding.

The cpSRP43 interaction data are of interest from several standpoints, the first of which relates to the extent and nature of the Lhcb1-cpSRP43 interaction. The crystal structure of cpSRP43 with bound L18 peptide revealed a complex in which 12 residues of the L18 peptide, corresponding to residues 156-167 within Lhcb1, were clearly defined within a groove in cpSRP43. Here, we have shown that Lhcb1 has close contact with cpSRP43 at positions 166, 169, 174 and 176 within Lhcb1, and this shows that the key area of interaction involves not just the L18 peptide, but also the first section of TM3 in Lhcb1 (see Fig. 1 for schematic of Lhcb1 membrane bound structure). Position 164, within the DPLG motif, did not produce any photoadducts, however this was to be expected as this position has previously been shown to be crucial for L18 binding [18]. Since the crosslinking data were obtained using a full-length Lhcb1 protein rather than a purified peptide, the outcomes were not constrained by the use of selected peptide regions. We are thus confident 
that cpSRP43 interacts with a relatively extended region of Lhcb1, comprising at least 20 residues from amino acids 156 - 176. As emphasised above, the experiments were conducted in aqueous buffer, and we believe that these data mirror the overall interaction that takes place in the chloroplast stroma.

While the structures of L18 residues 156-167 were well-resolved in the cpSRP43 crystal structure, we have no real information on the structure of the TM3 domain that we have now identified as interacting with cpSRP43. However, consideration of the available structural data suggests interesting possibilities. Stengel et al. [18] observed that the structure of cpSRP43bound L18 peptide resembled, quite closely, the structure of the same region of Lhcb1 in the membrane-bound mature state. In particular, it was noted that a quartet of residues, DPLG, curled around the cpSRP43 tyrosine 'hook' in a manner that closely resembled the way that these residues form around the hydroxyl group of the carotenoid molecule in the membrane bound Lhcb1 structure. This suggests that the cpSRP43-Lhcb1 binding process has evolved to mimic the normal interaction between Lhcb1 and the carotenoid molecule in the thylakoid-inserted form. This may have the advantage of allowing the Lhcb1 to pre-fold in a form that facilitates insertion with the correct topology and structure. Fig 6A shows the 3-dimensional structure of Lhcb1 with the L18 region indicated and the positions of the crosslinking residues (166-176) highlighted. Fig. 6B shows the structure of cpSRP43 with the bound L18 peptide (cyan) as determined by Stengel et al. [18] and TM3 shown modelled on the surface with the overall conformation maintained as in A. We propose that TM3 most likely binds in an additional groove on cpSRP43 as shown, and this would allow the four crosslinking sites to make contact with cpSRP43. The structure is shown rotated in Fig. 6C, which emphasises the discontinuous nature of the L18 and putative TM3 binding sites.

In the study by Stengel et al. [18] it was found that the Tyr204 'hook' on cpSRP43 was essential for binding of the L18 peptide; a Y204A mutant exhibited a massively-reduced binding affinity. While this pinpointed Tyr204 as a key component in the L18 binding reaction, we have shown in this study that the Y204A cpSRP43 mutant does crosslink to full-length Lchb1, albeit with lower efficiency. This suggests that the Y204A mutant binds Lhcb1 via additional interactions and possibly the TM3 residues identified in this study. A simplistic interpretation may be that the binding of Lchb1 to cpSRP43 involves recognition of two key determinants within Lhcb1: the L18 peptide (where interaction is dependent on Tyr204) and a second domain involving the first section of TM3. Further studies will be required to determine how and where this second domain binds on the cpSRP43 surface or whether this interaction is via an indirect binding involving cpSRP54.

As well as providing data on the extent to which cpSRP43 interacts with Lhcb1, the data have implications for the importance of this interaction. A notable feature of the crosslinking data is the observation that cpSRP43, on its own, is able to interact specifically with Lhcb1. This applies to all of the crosslinking adducts observed in this study (i.e. those located from residues 166176). Importantly, both the efficiency and the pattern of the crosslinking to wild type cpSRP43 are entirely unaffected by the presence or absence of cpSRP54. While cpSRP43 has been shown to have a novel chaperone activity, in the sense that it can reverse the aggregation of Lhcb1 $[26,27]$, this is the first demonstration that cpSRP43 is fully able to recognize and bind to insertion-competent, full-length Lhcb1 in the absence of its partner subunit. In turn, this suggests that cpSRP43 is a key component in the initial reaction by which cpSRP binds incoming 
substrates. Indeed, our data are consistent with the premise that cpSRP43 is effectively a key initial receptor for LHCP family proteins after import into the chloroplast. It is, however, important to stress that the role of cpSRP54 has yet to be characterised and this subunit may also play an important role in the binding of substrate.

\section{Funding}

This work was supported by Biotechnology and Biological Sciences Research Council grants to C.R., National Institutes of Health Grant GM26494 (AEJ) and by the Robert A. Welch Foundation (AEJ; Chair Grant BE-0017).

\section{References}

[1]. Luirink, J. and Sinning, I. (2004). SRP-mediated protein targeting: structure and function revisited. Biochim. Biophys. Acta, 1694, 17-35.

[2]. Driessen, A.J., and Nouwen, N. (2008). Protein translocation across the bacterial cytoplasmic membrane. Annu. Rev. Biochem. 77, 643-667.

[3]. Eichacker, L.A. and Henry, R. (2001) Function of a chloroplast SRP in thylakoid protein export. Biochim Biophys Acta, 1541, 120-134.

[4]. Kogata, N., Nishio, K., Hirohashi, T., Kikuchi, S. and Nakai, M. (1999). Involvement of a chloroplast homologue of the signal recognition particle receptor protein, Fts Y, in protein targeting to thylakoids. FEBS letts, 447, 329-333.

[5]. Li, X., Henry, R., Yuan, J., Cline, K. and Hoffman, N.E. (1995). A chloroplast homologue of the signal recognition particle subunit SRP54 is involved in the posttranslational integration of a protein into thylakoid membranes. Proc. Natl. Acad. Sci. USA, 92, 3789-3793.

[6]. Moore, M., Harrison, M.S., Peterson, E.C. and Henry, R. (2000) Chloroplast Oxa1p homolog albino3 is required for post-translational integration of the light harvesting chlorophyll-binding protein into thylakoid membranes. J. Biol. Chem. 275, 1529-1532.

[7]. Schuenemann, D., Gupta, S., Persello-Cartieaux, F., Klimyuk, V.I., Jones, J.D.G., Nussaume, L. and Hoffman, N.E. (1998). A novel signal recognition particle targets light-harvesting proteins to the thylakoid membranes. Proc. Natl. Acad. Sci. USA, 95, 10312-10316.

[8]. Kim, S.J., Jansson, S., Hoffman, N.E., Robinson, C. and Mant, A. (1999). Distinct "assisted" and "spontaneous" mechanisms for the insertion of polytopic chlorophyll-binding proteins into the thylakoid membrane. J. Biol. Chem. 274, 4715-4721.

[9]. Robinson, C., Thompson, S.J. and Woolhead, C. (2001) Multiple pathways used for the targeting of thylakoid proteins in chloroplasts. Traffic 2, 245-251. 
[10]. Schleiff, E. and Klosgen, R.B. (2001) Without a little help from 'my' friends: direct insertion of proteins into chloroplast membranes? Biochim Biophys Acta, 1541, 22-33.

[11]. Woolhead, C.A., Thompson, S.J., Moore, M., Tissier, C., Mant, A., Rodger, A., Henry, R. and Robinson, C. (2001) Distinct Albino3-dependent and -independent pathways for thylakoid membrane protein insertion. J. Biol. Chem. 276, 40841-40846.

[12]. DeLille, J., Peterson, E.C., Johnson, T., Moore, M., Kight, A. and Henry, R. (2000) A novel precursor recognition element facilitates posttranslational binding to the signal recognition particle in chloroplasts. Proc. Natl. Acad. Sci. USA, 97, 1926-1931.

[13]. Tu, C.J., Peterson, E.C., Henry, R. and Hoffman, N.E. (2000) The L18 domain of lightharvesting chlorophyll proteins binds to chloroplast signal recognition particle 43. J. Biol. Chem. 275, 13187-13190.

[14]. Groves, M.R., Mant, A., Kuhn, A., Koch, J., Dubel, S., Robinson, C. and Sinning, I. (2001). Functional characterization of recombinant chloroplast signal recognition particle. J. Biol. Chem. 276, 27778-27786.

[15]. Zhang, H., Wang, J. and Goodman, H.M. (1994) Expression of the Arabidopsis Gene Akr Coincides with Chloroplast Development. Plant Physiol, 106, 1261-1267.

[16]. Klimyuk, V.I., Persello-Cartieaux, F., Havaux, M., Contard-David, P., Schuenemann, D., Meiherhoff, K., Gouet, P., Jones, J.D., Hoffman, N.E. and Nussaume, L. (1999) A chromodomain protein encoded by the arabidopsis CAO gene is a plant-specific component of the chloroplast signal recognition particle pathway that is involved in LHCP targeting. Plant Cell 11, 87-99.

[17]. Sivaraja, V., Kumar, T.K., Leena, P.S., Chang, A.N., Vidya, C., Goforth, R.L., Rajalingam, D., Arvind, K., Ye, J.L., Chou, J., Henry, R. and Yu, C. (2005) Three-Dimensional Solution Structures of the Chromodomains of cpSRP43. J. Biol. Chem. 280, 41465-41471.

[18]. Stengel, K.F., Holdermann, I., Cain, P., Robinson, C., Wild, K. and Sinning, I. (2008) Structural basis for specific substrate recognition by the chloroplast signal recognition rarticle protein cpSRP43. Science 321, 253-256.

[19]. Wild, K., Halic, M., Sinning, I. and Beckmann, R. (2004) SRP meets the ribosome. Nat Struct Mol Biol, 11, 1049-1053.

[20]. Flanagan, J.J., Chen, J.C., Miao, Y., Shao, Y., Lin, J., Bock, P.E. and Johnson, A.E. (2003). Signal recognition particle binds to ribosome-bound signal sequences with fluorescence-detected subnanomolar affinity that does not diminish as the nascent chain lengthens. J. Biol. Chem. 278, 18628-18637. 
[21]. McCormick, P.J., Miao, Y., Shao, Y., Lin, J. and Johnson, A.E. (2003) Cotranslational protein integration into the ER membrane is mediated by the binding of nascent chains to translocon proteins. Molecular Cell, 12, 329-341.

[22]. Crowley, K.S., Reinhart, G.D. and Johnson, A.E. (1993) The signal sequence moves through a ribosomal tunnel into a noncytoplasmic aqueous environment at the ER membrane early in translocation. Cell, 73, 1101-1115.

[23]. Krieg, U.C., Walter, P. and Johnson, A.E. (1986). Photocrosslinking of the signal sequence of nascent preprolactin to the 54-kilodalton polypeptide of the signal recognition particle. Proc. Natl. Acad. Sci. USA, 83, 8604-8608.

[24]. Standfuss, J., Terwisscha, A.C., van Scheltinga, S., Lamborghini, M. and W Kuhlbrandt. 2005. Mechanisms of photoprotection and nonphotochemical quenching in pea light-harvesting complex at 2.5 A resolution. EMBO J. 24, 919-928.

[25]. High, S., Henry, R., Mould, R.M., Valent, Q., Meacock, S., Cline, K., Gray, J.C. and Luirink, J. (1997) Chloroplast SRP54 interacts with a specific subset of thylakoid precursor proteins. J. Biol. Chem. 272, 11622-11628.

[26]. Falk S, and Sinning, I. (2010). cpSRP43 is a novel chaperone specific for light-harvesting chlorophyll a,b binding proteins. J. Biol. Chem. In press.

[27]. Jaru-Ampornpan, P., Shen, K., Lam, V.Q., Ali, M., Doniach, S., Jia, T.Z. and Shan, S-O. (2010). ATP-independent reversal of a membrane protein aggregate by a chloroplast SRP subunit. Nat. Struct. Mol. Biol. 17, 696-702.

\section{Figure legends}

Figure 1. Overall structure of pea Lhcb1 and constructs used in this study. The upper diagram shows the overall structure of pea Lhbc1 with the transit peptide (TP), L18 peptide and 3 TM spans indicated. The TM2-L18-TM3 region has been expanded (lower diagram) to show the primary sequence and the positions of the crosslinkers used in this study (arrowheads). The L18 cpSRP-Lhcb1 interaction area, including the DPLG motif from the cpSRP43 structural study, is denoted by a dotted line, while a solid black line depicts the extended interaction area defined by the crosslinking data in this report.

Figure 2. Crosslinkers in Lhcb1 at positions 169 \& 176 react with cpSRP43 in the presence and absence of cpSRP54. Lhcb1 was synthesised with UV-activatable crosslinkers incorporated at positions 169 (within the L18 peptide) and 176 (within TM span 3) as detailed in Materials and Methods. After synthesis the translation product was incubated with buffer (-), cpSRP43 (43), the cpSRP43 $\Delta$ CD3 truncation (43A), cpSRP54 (54) or cpSRP43 + cpSRP54 combinations as indicated. After incubation, the crosslinker was activated by UV light and samples were analysed directly (upper panel) or after affinity purification of the cpSRP subunits using a Talon affinity matrix (lower panel). Crosslinking photoadducts generated with cpSRP43 are denoted X1 and 
$\mathrm{X} 2$; adduct $\mathrm{X} \Delta$ denotes crosslinking product with $\operatorname{cpSRP} 43 \Delta$. The three adducts are indicated by asterisks in the left panel. Mobilties of molecular mass markers (in kDa) are indicated on the left.

Figure 3. Crosslinking to cpSRP43 is dependent on UV illumination and the photo-reactive probe. Lhcb1 was synthesised with lysine at position 169 (D169K panel) or the photoactivatable ANB crosslinker (Lhc169) as indicated. After synthesis, the translation products were incubated with cpSRP54 and cpSRP43 $\Delta$ and samples were analysed directly (-) or after illumination with UV light (+UV) as indicated.

Figure 4. Interactions with cpSRP43 are clustered within L18 and TM3. Lhcb1 was synthesised with the ANB crosslinker in the positions indicated (152-176). After synthesis, the translation products were incubated with buffer (-), cpSRP43 (43) or cpSRP43 $\Delta$ CD3 (43 $\Delta$ ). The reactions were then illuminated with UV light and samples were analysed directly (total) or after affinity purification of the SRP subunits using Talon resin (lower panel). Labels as in Figure 2.

Figure 5. A tyrosine 'hook' on cpSRP43 is required for efficient crosslinking to Lhcb1. Lchb1 with the ANB crosslinker at position 169 was incubated with buffer (-), cpSRP43 (43) or the Y204A mutated version of cpSRP43 (43*), alone or with cpSRP54, or the cpSRP43 $\Delta$ CD3 mutant $(43 \Delta)$. The reactions were then illuminated with UV light and samples were analysed directly. The major, upper crosslink to cpSRP43 is denoted by X1, with the adduct in the Y204A samples is denoted by an arrowhead. Other labels as in Figure 2.

Figure 6. Model for the interaction of Lhcb1 with cpSRP43. Image A shows the 3-dimensional structure of membrane bound Lhcb1 with the L18 peptide indicated and the additional cpSRP43 interaction sites circled. Crosslinked residues (166, 169, 174 and 176) are indicated in red. Other residues analysed as potential crosslinking sites in this study are coloured orange. Image B shows the structure of cpSRP43 with Lhcb1 residues 156-167 of the L18 peptide (L18p, cyan, PDB: 3DEO) bound within a groove as determined by Stengel et al. (2008). Note that the DPLG motif curls around Tyr204 on the cpSRP43 surface. The Lhcb1 TM3 region is shown positioned in an adjacent groove on the surface of cpSRP43 after superposition of the L18-TM structure from the native structure (yellow, PDB: 2BHW, Standfuss et al, 2005). The positions of the crosslinking sites (residues 166, 169, 174 and 176) are denoted by red colouring of side chains. C: as B with the image rotated by $90^{\circ}$. The position of Y204 is indicated by the asterisk. 


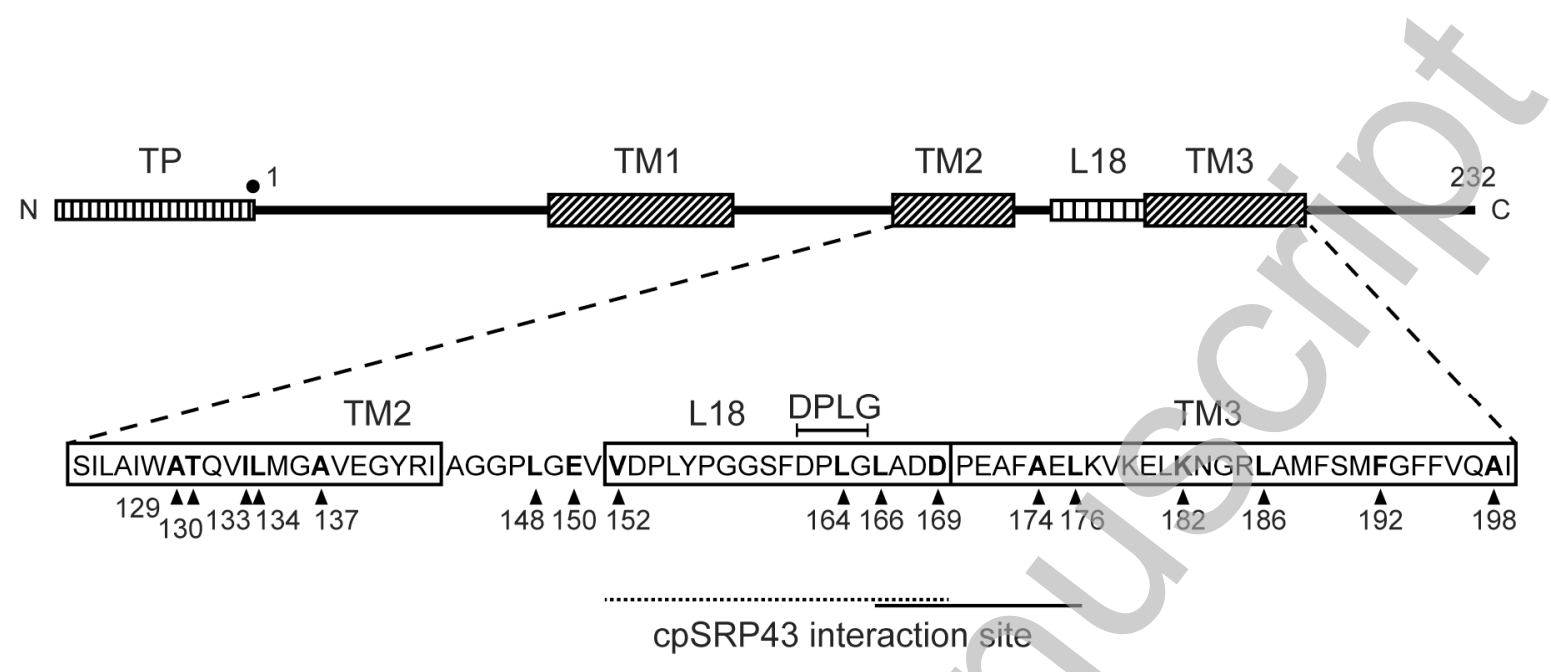

\section{Figure 1}




kDa \begin{tabular}{llllll} 
& \multicolumn{6}{c}{ Lhc169 } \\
\cline { 2 - 5 } & 43 & $43 \Delta$ & 54 & $43 / 54$ & $43 \Delta / 54$
\end{tabular}

\begin{tabular}{llll} 
Lhc176 & \\
\hline$-43 \quad 43 \Delta$ & 54 & $43 / 54$ & $43 \Delta / 54$
\end{tabular}
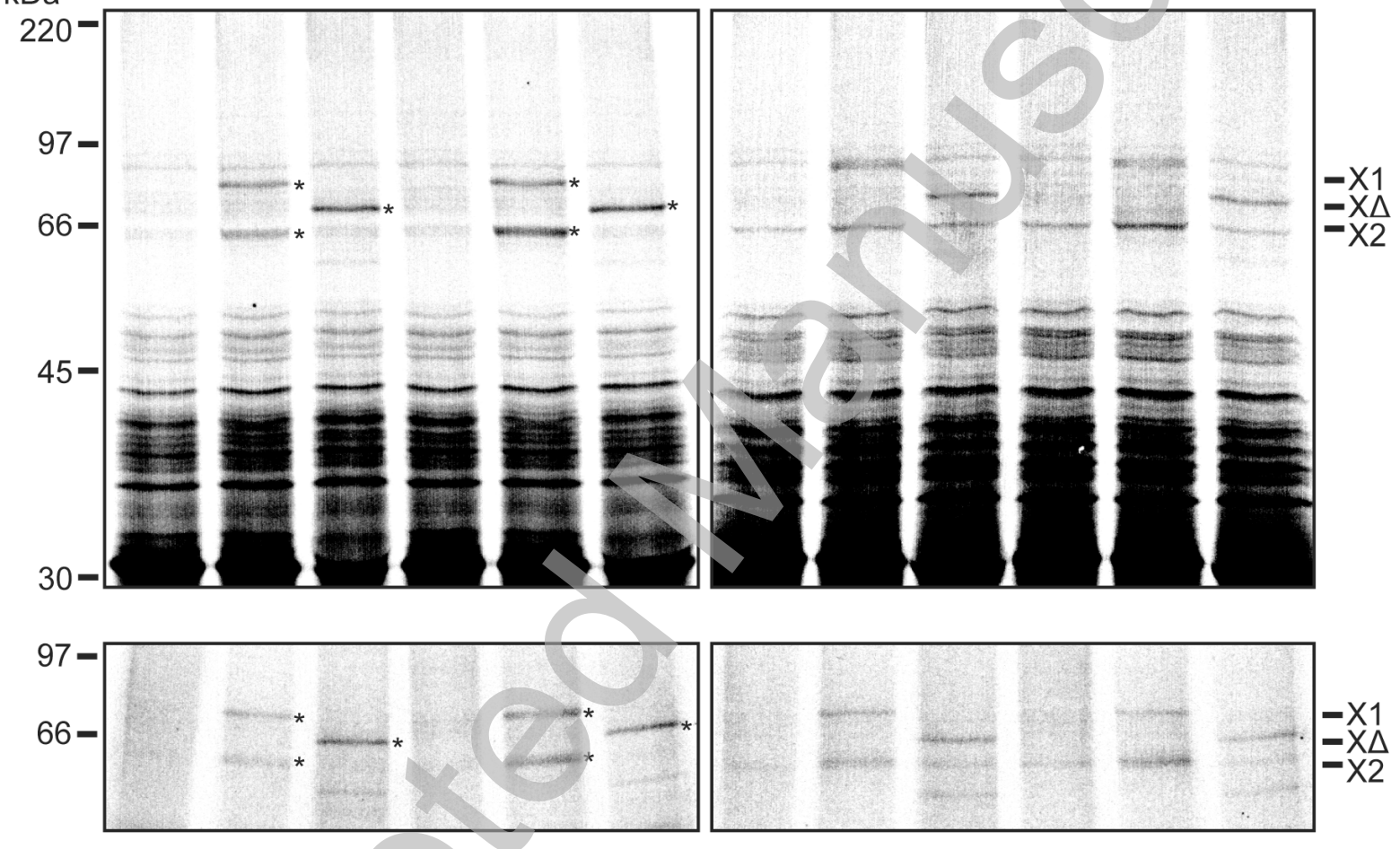

Figure 2 


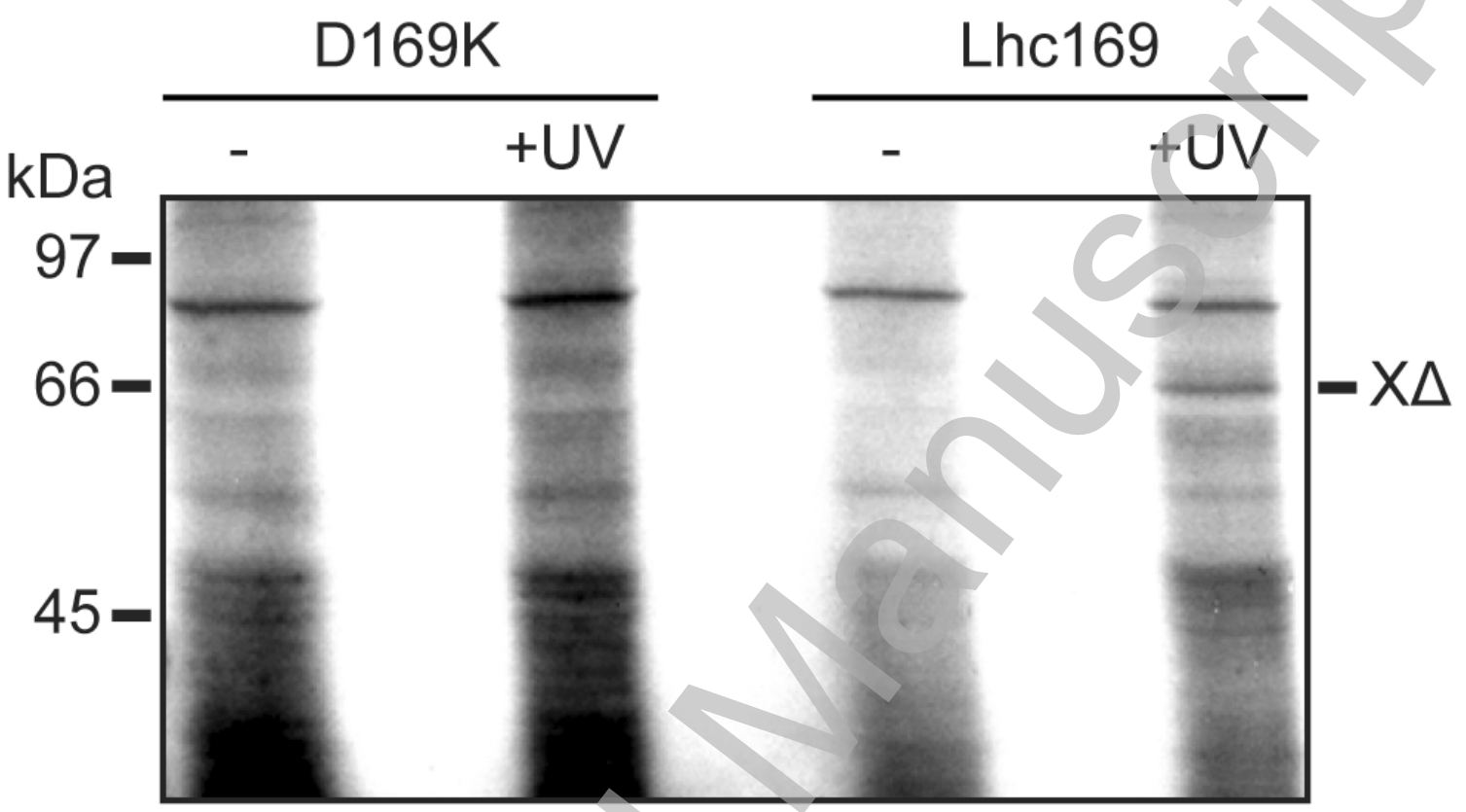

Figure 3 


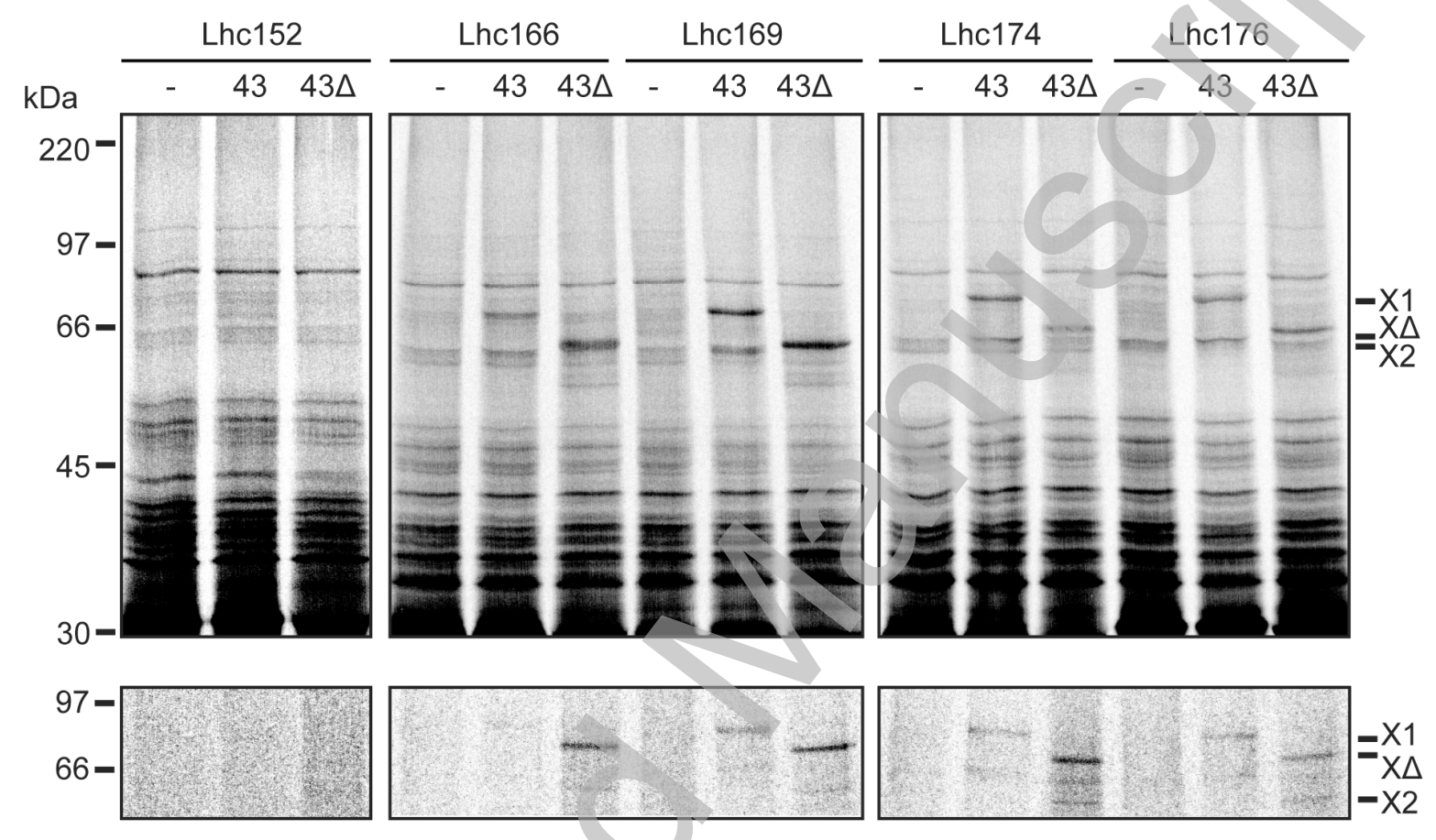

Figure 4 


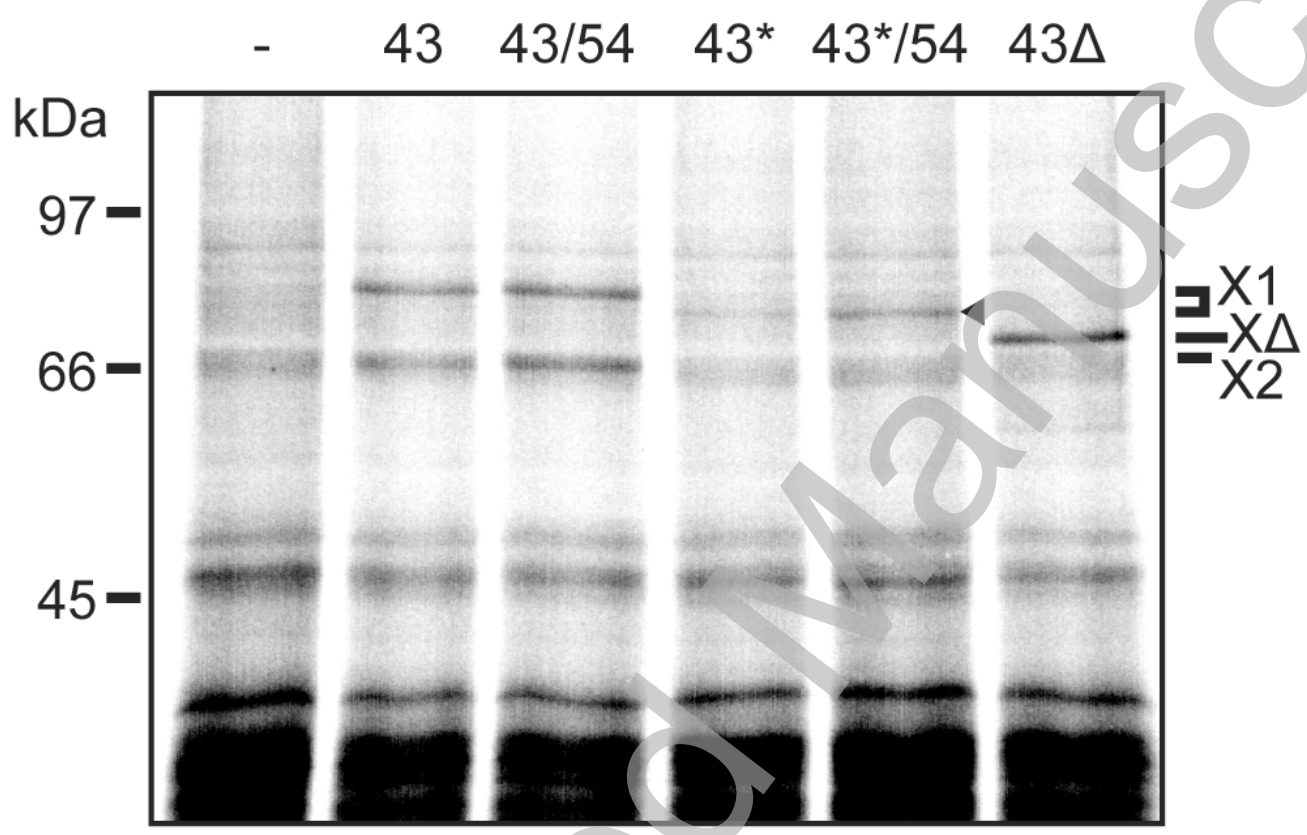

Figure 5 
A
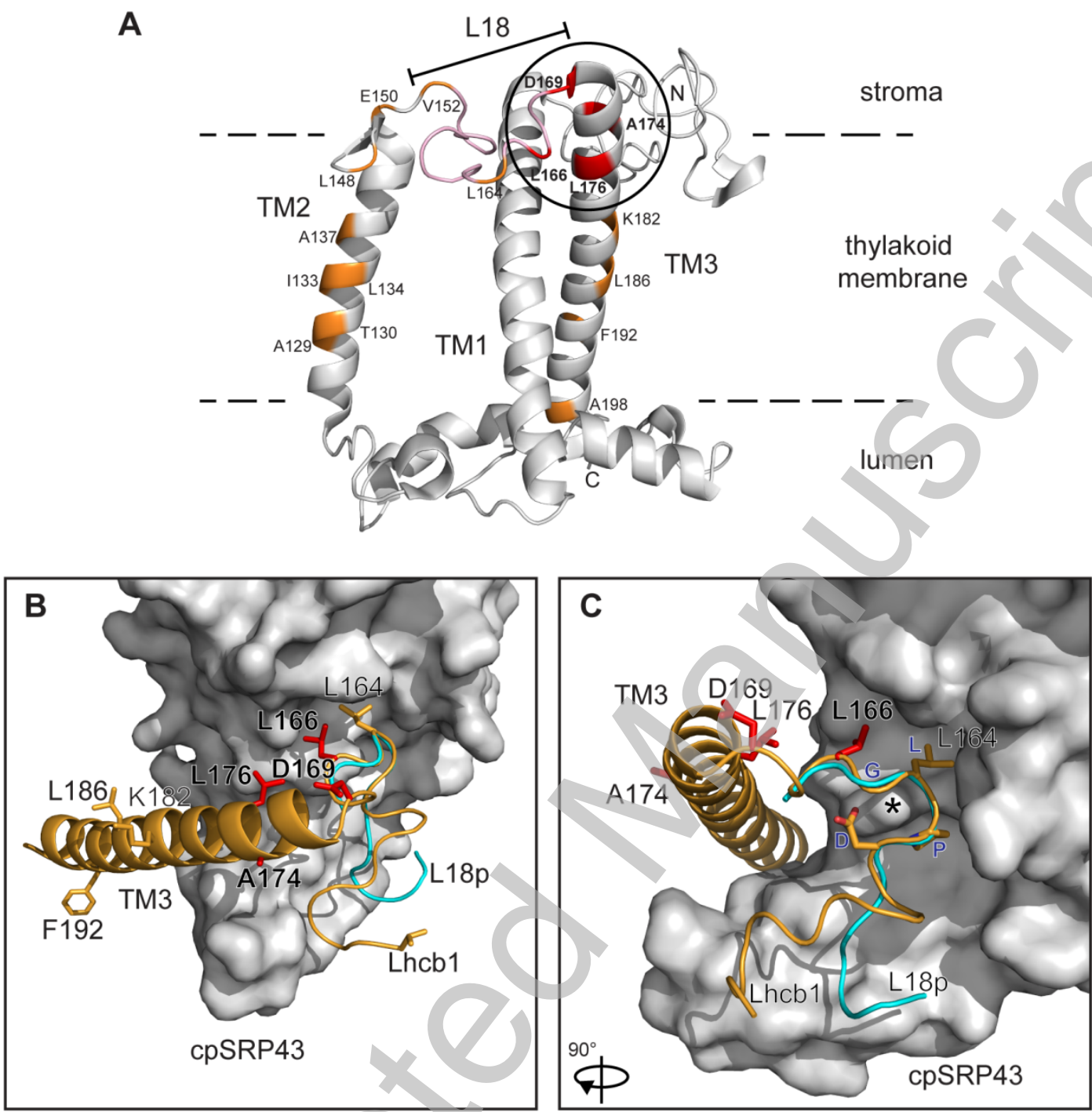

Figure 6 